\title{
ENHANCEMENT IN FATIGUE CHARACTERISTICS BY ELASTO-PLASTIC ANALYSIS OF PRE-STRESSED HOLES
}

\author{
Gopalakrishna H.D. ${ }^{1}$, Krishna M. ${ }^{2}$, Pradeep R. ${ }^{3}$, Janaki R. ${ }^{4}$ \\ ${ }^{1,2,3,4}$ Department of Mechanical Engineering, Rashtriya Vidhyalaya College of Engineering, \\ Bangalore, India \\ Email: 'hurligopal@yahoo.co.uk
}

\begin{abstract}
The holes made to accommodate the fasteners in many of the engineering applications as in aircraft structures are subjected to cyclic loads. The improvement in fatigue characteristics by the cold expansion process is the established practice. It has been the ongoing research trend to quantify the enhancement in the fatigue strength due to the induction of residual stresses by prestressing the drilled hole. The optimization of the fatigue strength and to establish corresponding percentage of expansion of the fastener hole is the focus of this paper. Expanding into Taylor series, the functional relation between cyclic stress and mean stress given by Soderberg and subsequent application of interface theory lead to the formulation of the objective function. The expansion is linear in the elastic zone governed by Hooke's law and non-linear in plastic zone obeying the Holloman's law. The elasto-plastic relation between stress and strain provides the constraint condition. The non-linear relation of optimization is solved by a novel method to get the optimum enhancement fatigue strength due to cold expansion of the hole. The analytical results are compared with the experimental analysis for justification as reported in previous literature.
\end{abstract}

Key words: Elasto-plastic analysis, Cold hole expansion, Fatigue life improvement.

\section{INTRODUCTION}

Variations in aerodynamic forces induce fatigue stresses in aircraft structures. The fabricated sheet metal constructions are joined by riveted fasters. The rivet holes not only increase the stress concentration, also result in decrease of fatigue strength. The development of fatigue cracks leads to failure of structures. Fastener holes being the weakest zones are responsible for crack initiation. It has been well established that work hardening mechanism can improve the fatigue characteristics of metallic elements. The cold expansion of the fastener holes induces the residual stresses in the vicinity of the hole resulting in betterment of fatigue behaviour of the material of construction. Aircraft fabrication generally makes use of aluminium or aluminium alloys as they are light in weight. In diversified applications the mild steel is also one of the materials used.

The latest developments reported in [1-3] focus on the following aspects. An experimental work in [1] describes the variation of fatigue life of the fastener holes subjected to cold expansion influenced by the cyclic variation in stresses. The data of [1] has been referred as basis for the analytical study of this work that predicts the enhancement in endurance strength, in reversed loading of cold expanded hole in aluminium alloy and mild steel specimens. The reports in [2] emphasize on the finite element analysis to predict the enhancement in fatigue life of the cold expanded stop holes.
The properties of these materials like Young's modulus, plastic co-efficient, work hardening index and the endurance limit, are obtained from the literatures, established through standard experimentation. The effort of this paper concentrates on development of an analytical model to predict the enhancement in fatigue strength of aluminium and mild steel in the process of pre-stressing due to cold expansion of the hole. The work hardening effect of cold expansion leaves compressive residual stresses expected to facilitate the dynamic load withstanding capability. To quantify the effect, the stressstrain relation of the material, and Soderberg's relation between variable and mean stresses are the initial take off steps in this analysis.

The experimental evidences of Goodman and Soderberg estimate a functional relation between the variable stresses and mean stress. For a small change in mean stress change in variable stress can be given by Taylor series expansion. Neglecting the contribution of higher order terms and applying interface theory (briefly explained in the appendix) the variable stress is given the expression for objective function of optimization.

The mechanism of cold expansion is a complex combination of elastic and plastic compression. The stress-strain relation is linear in the elastic zone following the well known Hooke's law [3]. But $\sigma-\varepsilon$ plot exhibits nonlinearity in the plastic zone explained by Holloman's law [4]. The elasto-plastic behaviour gets formulated to provide constraint condition. The solutions of constraint condition quantify the elastic and plastic stress 
components that would maximize the enhanced fatigue stress by effect of cold expansion.

The prime objective is to track the variation in fatigue strength for varying expansion and to locate the optimality condition for the given material. This establishes the criteria for percentage expansion for optimal performance. The variation in expansion pressure for different percentage of expansions gives an input to the finite element analysis.

The objectives of this paper is to solve the elastoplastic stress-strain relation using newly developed interface theory and to predict analytically the improvement in fatigue strength basing the development on the Soderberg's relation [3] and Taylor series expansion [5]. The results thus obtained are compared with the experimental data [1] for the justification. The material properties cited in [6] are used to carryout the numerical analysis on the specimens made of aluminium alloy and mild steel.

\section{FORMULATION}

\section{A. Elasto-plastic Analysis}

The cold expansion of the circular hole as shown by Fig-1, exhibit a elasto-plastic behavior, of compression taking place radially. The elastic behavior is governed by the Hooke's law relating stress linearly with the strain. The non-linear relation between stress and strain because of work hardening effect is explained by Hollomann's law of plasticity as cited in [6]. Hence the stress induced due to cold expansion can be written as

$$
\sigma=E \epsilon_{e}+K\left(\epsilon_{p}\right)^{n}
$$

Where, $\epsilon_{\mathrm{e}}$ and $\epsilon_{\mathrm{p}}$ are the elastic and plastic components of strain,

$\mathrm{E}$ is modulus of elasticity, $\mathrm{K}$ is the plastic co-efficient and $\mathrm{n}$ is the work hardening index.

The solution to equation (1) can be from interface theory,

$$
\begin{aligned}
& \begin{array}{l}
\epsilon^{*}=\frac{\mathrm{K}}{\mathrm{Q}} \quad \text { gJq } \quad \epsilon^{\mathrm{b}}=\left[\left(\mathrm{I}-\frac{\mathrm{K}}{\mathrm{E}}\right) \frac{\mathrm{X}}{\mathrm{C}}\right]_{\frac{\mathrm{T}}{\mathrm{T}}} \\
\text { Or }
\end{array} \\
& \epsilon_{c}=\left[\left(1-\frac{K}{E}\right) \frac{\sigma}{E}\right] \text { and } \quad \epsilon_{p}=\left[\frac{\sigma}{K}\right]^{\frac{1}{n}}
\end{aligned}
$$

$E$ being greater than $K, \epsilon_{p}$ in solution (2) returns a negative value hence can be considered to be trivial. Hence the analysis is based on solution (3). The total percent of expansion can now be

$$
\epsilon_{e}+\epsilon_{P}=\left[\left(1-\frac{K}{E}\right) \frac{\sigma}{E}\right]+\left(\frac{\sigma}{K}\right)^{\frac{1}{n}}
$$

Varying $\sigma$ in (4) it is possible to estimate the expansion that occurs in the process. Fig. 2 and Fig. 3 show the variation of $\epsilon_{\mathrm{e}}$ and $\epsilon_{\mathrm{p}}$ with respect to $\sigma$ applied stress, respectively. The values of $K, n$ and $E$ as taken from [5] are tabulated in Table -1 .

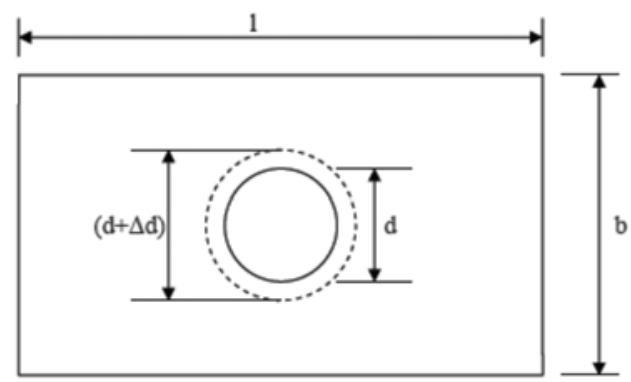

Fig. 1. Specimen sketch

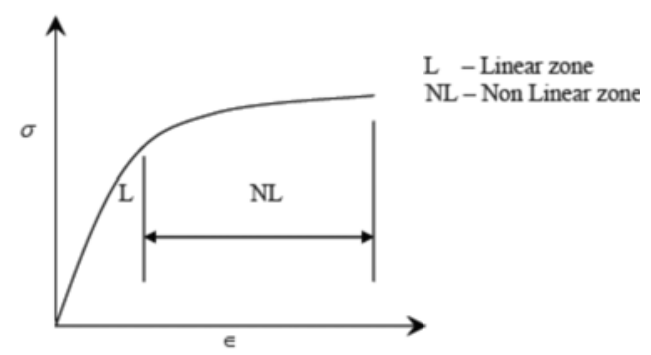

Fig. 2(a). Typical $\sigma-€$ curve

\section{B. Prediction of enhancement in endurance strength}

Soderberg and Goodman [3] established experimentally a functional relation between mean stress, $\mathrm{m} \sigma$ and variable stress, $\mathrm{f} \sigma$ in cyclic loading. The plotted result can be represented by the function,

$$
\sigma_{f}=f\left(\sigma_{m}\right)
$$

The increment in $\mathrm{f} \sigma$ due to increment in $\mathrm{m} \sigma$ can be expressed by Taylor series expansion as

$$
f\left(\sigma_{m}+\Delta \sigma_{m}\right)=f\left(\sigma_{m}\right)+\frac{\partial f}{\partial \sigma_{m}} \Delta \sigma_{m}+\ldots \ldots \ldots \ldots \ldots \ldots \ldots . . \frac{\partial^{n} f\left(\Delta \sigma_{m}\right)^{m}}{\partial \sigma_{m} m !}
$$


Where,

$m$ is the order of the PDE (6).

Theequation (6) can be written as,

$$
\frac{\Delta \sigma_{f}}{\Delta \sigma_{m}}=\frac{\partial \sigma_{f}}{\partial \sigma_{m}}+\frac{\partial^{2} \sigma_{f}}{\partial \sigma_{m}{ }^{2}} \frac{\Delta \sigma_{m}}{2}+\ldots \ldots . .+\frac{\partial^{m} \sigma_{f}}{\partial \sigma_{m}} \frac{\left(\Delta \sigma_{m}\right)^{m-1}}{m !}
$$

For the condition of minimization (lower bound values),

$$
\frac{\partial \sigma_{f}}{\partial \sigma_{y}}=\frac{\Delta \sigma_{m}}{\sigma_{y}}\left(\frac{m !}{\left(\Delta \sigma_{m}\right)^{m-1}}\right)=\frac{\Delta \sigma_{f}}{\Delta \sigma_{m}}
$$

This implies that,

$$
\Delta \sigma_{m}=\sqrt[m-\sqrt[2]{m} !]{ }
$$

The selection of $m$ determines the variation of mean stress. From the Soderberg relation for ductile material as in [3]

$$
\frac{1}{N}=\frac{\Delta \sigma_{m}}{\sigma_{y}}+K \frac{\Delta \sigma_{f}}{\sigma_{e n}}
$$

This leads to

$$
\Delta \sigma_{f}=\left(\frac{1}{N}-\frac{\Delta \sigma_{m}}{\sigma_{y}}\right) \frac{\sigma_{e n}}{K}
$$

The value of $\Delta \sigma_{m}$ is computed from the following equation,

$$
\Delta \sigma_{m}=\Delta \sigma_{e}+\Delta \sigma_{p}
$$

\section{Table 1. Material properties}

\begin{tabular}{|l|l|l|c|c|}
\hline Material & $E(G \mathrm{pa})$ & $K(\mathrm{Mpa})$ & $\|$ & $\sigma_{a}$ (Mpa) \\
\hline Al alloy & 69.00 & 570.00 & 0.347 & 68.5 \\
\hline Mild steel & 206.00 & 567.00 & 0.264 & 83.5 \\
\hline
\end{tabular}

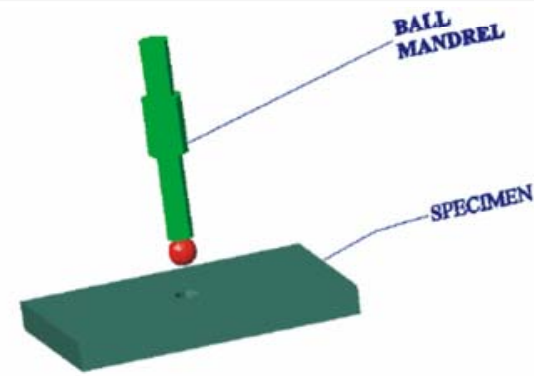

Fig. 2(b). Specimen and Ball Mandrel

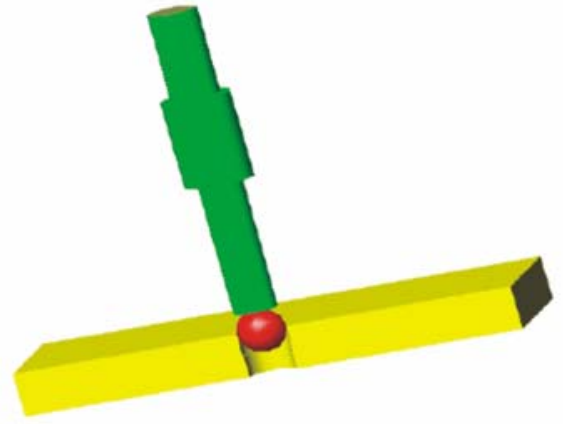

Fig. 2(c). Cold expansion process

\section{Implementation}

The equations derived are used to compute the stresses in the elastic and plastic regions and to determine the fatigue stress. The values of elastic modulus, plastic coefficient, work hardening index for materials considered like Aluminium and Mild steel are selected from the design

\begin{tabular}{|c|c|c|c|c|c|}
\hline Material & $\begin{array}{c}\text { Percentage of } \\
\epsilon=\epsilon,+\epsilon, \\
\%)\end{array}$ & $\begin{array}{c}\text { Change in } \\
\text { elastic stress } \\
\Delta \sigma_{e_{1}, \mathrm{MPa}}\end{array}$ & $\begin{array}{l}\text { Change in } \\
\text { plastic stress } \\
\Delta \sigma_{p, M P a}\end{array}$ & $\begin{array}{l}\text { Change in } \\
\text { mean stress } \\
\Delta \sigma_{m, M P a}\end{array}$ & $\begin{array}{c}\text { Change in } \\
\text { fatigue stress, } \\
\Delta \sigma_{f} \mathrm{MPa}\end{array}$ \\
\hline \multirow{4}{*}{$\mathrm{Al}$ alloy } & 1 & 0.090 & 0.092 & 0.182 & 0.0481 \\
\hline & 2 & 0.180 & 0.119 & 0.300 & 0.0478 \\
\hline & 3 & 0.271 & 0.138 & 0.409 & 0.0477 \\
\hline & 4 & 0.312 & 1.53 & 0.514 & 0.0476 \\
\hline \multirow{4}{*}{ Mild steel } & 1 & 0.053 & 0.191 & 0.244 & 0.0587 \\
\hline & 2 & 0.106 & 0.229 & 0.335 & 0.0586 \\
\hline & 3 & 0.156 & 0.255 & 0.411 & 0.0585 \\
\hline & 4 & 0.213 & 0.292 & 0.488 & 0.0584 \\
\hline
\end{tabular}
data hand book.

\section{RESULTS AND DISCUSSION}

\subsection{Fatigue life prediction with mean stress variation}

Table 2. Variation of Stresses with increase in expansion

Comparative plot of various stresses for Aluminium and Mild Steel

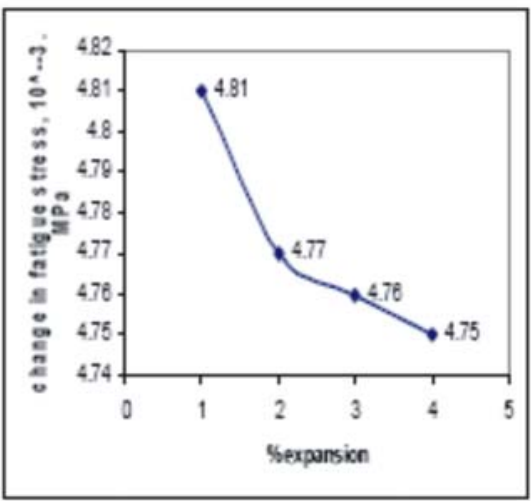

Fig. 3(a). Change in fatigue stress vs. \% expansion- Al 


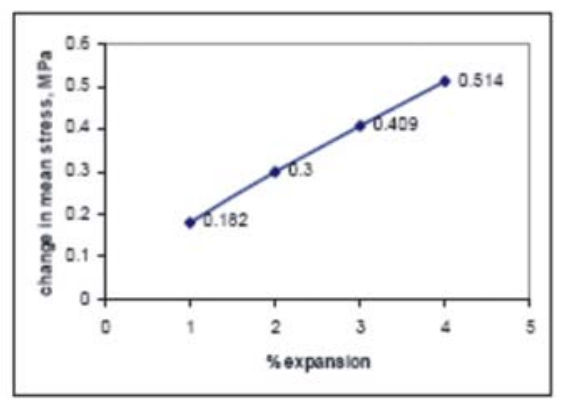

Fig. 3(b). Change in mean stress vs. \% expn- Al

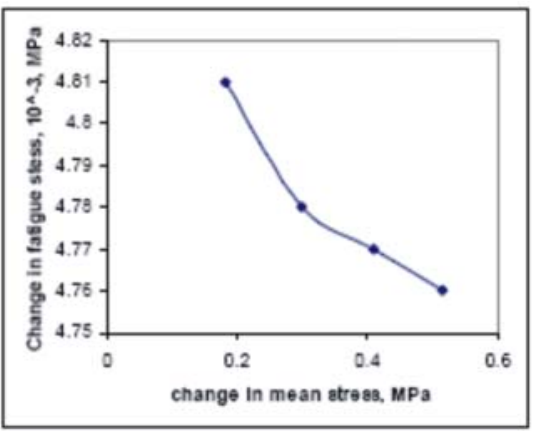

Fig. 3(c). $\Delta$ mean stress vs. $\Delta$ fatigue stress-Al

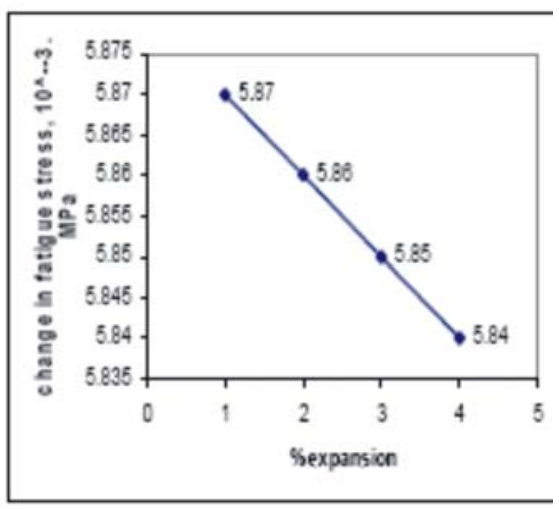

Fig. 3(d). Change in fatigue stress vs. \% expansion- MS

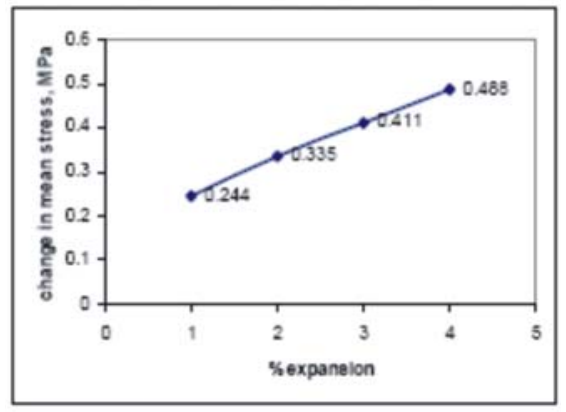

Fig. 3(e). Change in mean stress vs. \% expn- MS

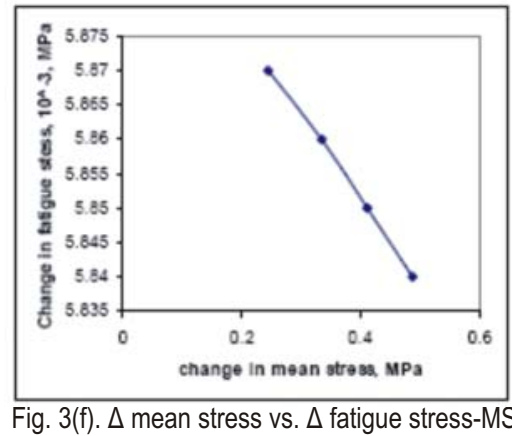

IV. CONCLUSION

Analytical model has been developed to represent the cold expansion process. The elasto-plastic equation representing the behaviour of cold expansion is solved and the mean stress for different percentages of expansion is obtained. The model developed to predict the enhancement in fatigue strength gave the variations with respect to mean stress due to cold expansion. The lower bound on the fatigue life was considered to be optimistic.The analytical result closely matches with the experimental data reported in previous literature. Experimental verification may be performed to compare the results with the analytical model developed.

\section{REFERENCES}

[1] Callinan R.J. and Wang, C.H., 1999, "On the fatigue enhancement of interference fitted stop drilled holes", International .Journal of fatigue, Elsevier, 21, pp, 865-872

[2] Hall, Holowenko and Laughlin, 1983, "Machine Design", Singapore, Mc-Graw Hill

[3] Pourboghrat,F. and Chu E, 1995, " Spring back in plain strain/stretch/draw sheet forming", International .Journal .Mechanical Science, Vol 36, No 3, pp,327-341

[4] E. Kreyzig, "Advanced Engineering Mathematics", $8^{\text {th }}$ edition.

[5] Minguez J.M. and Vigwell J, 2005, "Fatigue life of an aerospace aluminium alloy subjected to cold expansion and cyclic temperature regime", Engineering failure analysis, Elsevier.

[6] K Mahadevan, K Balaveera Reddy, 1987, " Design Data Hand Book", New Delhi, CBS Publishers

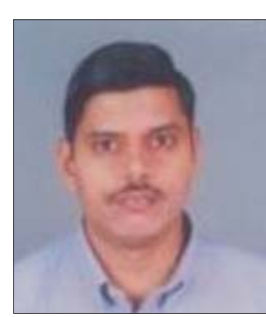

Mr. H. D. Gopalakrishna is a Research Scholar at VTU, Belgaum and an Assistant Professor at the Department of Mechanical Engineering at Rashtriya Vidyalaya College of Engineering, Bangalore. He obtained his Masters degree in Manufacturing Engineering from San Jose State, CA, USA and his research interests are Manufacturing Science and Materials. 